\title{
Effects of Transplacental Dexamethasone Therapy on Fetal Immune-Mediated Complete Heart Block
}

\author{
Mika Saito ${ }^{a}$ Earl Silverman ${ }^{b} \quad$ Fraser Golding $^{a} \quad$ Vitor Guerra $^{a} \quad$ Linda Hiraki $^{b}$ \\ Varsha Thakur ${ }^{\mathrm{a}}$ Edgar Jaeggi ${ }^{\mathrm{a}}$ \\ ${ }^{a}$ Department of Pediatrics, Fetal Cardiac Program, Labatt Family Heart Centre, The Hospital for Sick Children, \\ University of Toronto, Toronto, ON, Canada; ' Division of Rheumatology, The Hospital for Sick Children, \\ University of Toronto, Toronto, ON, Canada
}

\section{Keywords}

Fetal echocardiography · Prenatal treatment · Complete heart block-steroids

\begin{abstract}
Introduction: Antibody-mediated complete atrioventricular block (CAVB) is considered irreversible. We sought to examine the effects of transplacental steroids on fetal AV conduction. Methods: Fifty-nine fetuses diagnosed with CAVB at our center from 1996 to 2018 were reviewed. Routine dexamethasone administration to birth was used to limit cardiac inflammatory damage. Restoration of fetal AV conduction was classified as "unexpected" treatment response. Results: CAVB resolved in 5/29 (17\%) fetuses first treated $\leq 24$-week gestation with $8 \mathrm{mg} /$ day of dexamethasone, when compared with $0 / 30(0 \%)$ when treatment was initiated later and/or at a starting dose of $4 \mathrm{mg} /$ day (odds ratio 13.69; $95 \%$ confidence interval $0.72-260.13 ; p=0.024)$. Treatment response was also associated with a faster ventricular rate at diagnosis (median [range]: 80 [60-97] beats per minute [bpm] vs. 58 [38-92] bpm; $p=0.0036)$. CAVB reappeared in all 5 responders either prenatally $(n=1)$ or postnatally before
\end{abstract}

karger@karger.com www.karger.com/fdt

Karger $\stackrel{\text { ' }}{=}$

GOPEN ACCESS
(C) 2021 The Author(s)

Published by S. Karger AG, Basel

This is an Open Access article licensed under the Creative Commons Attribution-NonCommercial-4.0 International License (CC BY-NC) (http://www.karger.com/Services/OpenAccessLicense), applicable to the online version of the article only. Usage and distribution for commercial purposes requires written permission. $(n=3)$ or after $(n=1)$ the first year of life. When compared with infants with treatment-resistant CAVB (median followup 10.3 years), responders (median follow-up 12.3 years) required postnatal pacing less frequent (2/5 [40\%] vs. 45/49 [92\%]; $p=0.013)$. Conclusions: In a subgroup of CAVB fetuses, dexamethasone transiently restored AV conduction. This was associated with a lower rate of postnatal pacing when compared with nonresponders.

(c) 2021 The Author(s)

Published by S. Karger AG, Basel

\section{Introduction}

Isolated complete atrioventricular block (CAVB) is predominantly associated with the fetal exposure to hightiter anti-Ro antibodies [1]. These maternal antibodies cross the placenta and, in the susceptible fetus, may trigger inflammation, necrosis, and fibrosis of the AV node $[2,3]$, which commonly manifests as sudden-onset CAVB between 18 and 24 gestation weeks. Development of CAVB may be preventable if the inflammation is detected and treated with transplacental steroids at an early disease state of incomplete first- or second-degree heart block [4, 
5]. Restoration of 1:1 AV conduction is not expected once AV nodal conduction is completely blocked $[4,6]$. In this situation, transplacental anti-inflammatory medication has primarily been advocated to treat or prevent antibody-mediated disease of the working myocardium [7]. The aims of this study of fetuses with CAVB were to determine (a) short- and long-term effects of perinatal steroids on the AV conduction system and (b) if conduction improved, to identify factors associated with improvement in AV conduction.

\section{Methods}

The Review Ethics Board of the Hospital for Sick Children (IRB) approved this retrospective study (\#1000011904). The need of written parental consent of patient enrollment was waived by the IRB.

\section{Patients}

Included in this retrospective study were all 59 fetuses that received transplacental steroids for immune-mediated CAVB from January 1996 to December 2018. Eligible cases were identified from our Fetal Cardiac Program database. The presence of maternal anti-Ro antibodies was confirmed in all cases by solid-phase enzyme-linked immunosorbent assay or chemiluminescence immunoassay at time of heart block diagnosis $[1,5]$. Treatment recommendations included the maternal administration of (1) oral dexamethasone from CAVB diagnosis $(8 \mathrm{mg} /$ day for 2 weeks, 4 $\mathrm{mg}$ /day to $28-30$ weeks, and $2 \mathrm{mg}$ /day to birth), (2) oral salbutamol (30-40 mg/day) for fetal bradycardia $<50$ beats per minute $(\mathrm{bpm})$, and (3) intravenous immune globulin (IVIG; $1 \mathrm{~g} / \mathrm{kg}$ or maximum $70 \mathrm{~g} /$ dose every 2-3 weeks, single neonatal dose of $2 \mathrm{~g} / \mathrm{kg}$ ) if increased cardiac echogenicity suggestive of endocardial fibroelastosis (EFE) was diagnosed. Prior to 2005, the recommended starting dose of dexamethasone was $4 \mathrm{mg} /$ day [7]. Reflecting the treatment modification, most $(15 / 20 ; 75 \%)$ fetuses before 2005 were started on $4 \mathrm{mg} /$ day, while $36 / 39$ (92\%) subsequent cases received $8 \mathrm{mg} /$ day for the first 2 weeks of treatment.

\section{Methods}

Serial fetal echocardiograms to birth $(n=56)$ or in utero demise $(n=3)$ were obtained in all cases. Fetal atrial (A) and ventricular (V) rates and AV chronology were assessed from simultaneous superior vena cava/aorta Doppler flow and atrioventricular MMode tracings, respectively [5]. Doppler-derived AV duration was measured if there was $1: 1 \mathrm{AV}$ conduction and compared with published reference values [8]. Diagnosis of CAVB was defined by the complete absence of AV conduction with independent atrial and ventricular rhythms. Resolution of CAVB with treatment to firstdegree AV block (AVB I), second-degree AV block (AVB II), or normal AV conduction was classified as "unexpected" treatment response. The diagnosis of AVB II was based on the intermittent failure of AV conduction. In type-1 AVB II, the nonconducted atrial event was preceded by conducted beats that displayed progressive AV interval (AVI) lengthening, while the AV duration did not change in type-2 AVB II. 1:1 AV conduction with AVI $>3 \mathrm{z}$ scores was considered prolonged and defined as AVB I. Adminis- tration of a single neonatal dose of IVIG $(2 \mathrm{~g} / \mathrm{kg})$ and prednisone for 2-3 months was advised if improved AV conduction or EFE persisted to birth. AHA/ACC Class I indications of CAVB were used as indications for permanent pacing $[9,10]$. Collected patient data included gestational age of CAVB diagnosis, longitudinal echocardiographic and electrocardiographic findings, treatment, and outcome to December 2019.

\section{Statistical Analysis}

Data are expressed as mean with standard deviation, median (range), or proportions as appropriate. Baseline clinical and echocardiographic findings were compared among fetal cohorts with versus without a treatment response. Fisher's exact test was used for categorical variables and Student's $t$ test or Mann-Whitney U test for continuous variables. Kaplan-Meier estimates and log rank were used to plot freedom from pacemaker implantation and to assess between-cohort differences. Data were analyzed with Graph Pad Software for Windows (Graph Pad Inc., San Diego, CA, USA).

\section{Results}

Table 1 shows the findings of 59 study cases without (nonresponders) versus with (responders) improvement in AV conduction on anti-inflammatory treatment. Patients diagnosed $\leq 24$ gestation weeks were significantly more likely to receive $8 \mathrm{mg} /$ day of dexamethasone when compared with a later CAVB diagnosis $(p=0.0037)$.

\section{Prenatal Effects}

CAVB persisted in 54/59 (92\%) cases. This includes $30 / 30$ fetuses that received dexamethasone after 24 gestation weeks and/or at a starting dose of $4 \mathrm{mg} /$ day (group 1). In contrast, restoration of AV conduction was documented in $5 / 29$ (17\%) fetuses treated for the first 2 weeks with $8 \mathrm{mg} /$ day of dexamethasone and $\leq 24$ gestation weeks (group 2) (group 2 vs. 1: odds ratio 13.69; 95\% confidence interval $0.72-260.13 ; p=0.024)$. Reversal of CAVB was furthermore significantly associated with a faster ventricular rate (VR) at the time of CAVB diagnosis, while other findings were largely comparable among the cohorts.

\section{Outcome of Responders}

Figure 1 illustrates the prenatal and postnatal evolution of AV block of the 5 responders. CAVB reappeared in all of them either prenatally $(n=1)$ or postnatally before $(n=3)$ or after $(n=1)$ the first year of life:

Case 1 has been previously reported [11]. Dexamethasone was started following CAVB diagnosis (VR $80 \mathrm{bpm}$ ) at $21+4$ weeks gestation. AV conduction improved to type-1 AVB II at $22+3$ weeks, AVB I (AVI $170 \mathrm{~ms}$ ) at 23 +4 weeks, and normal 1:1 conduction (AVI $140 \mathrm{~ms}$ ) at 27 weeks. The AV conduction deteriorated to 2:1 AVB at 29 


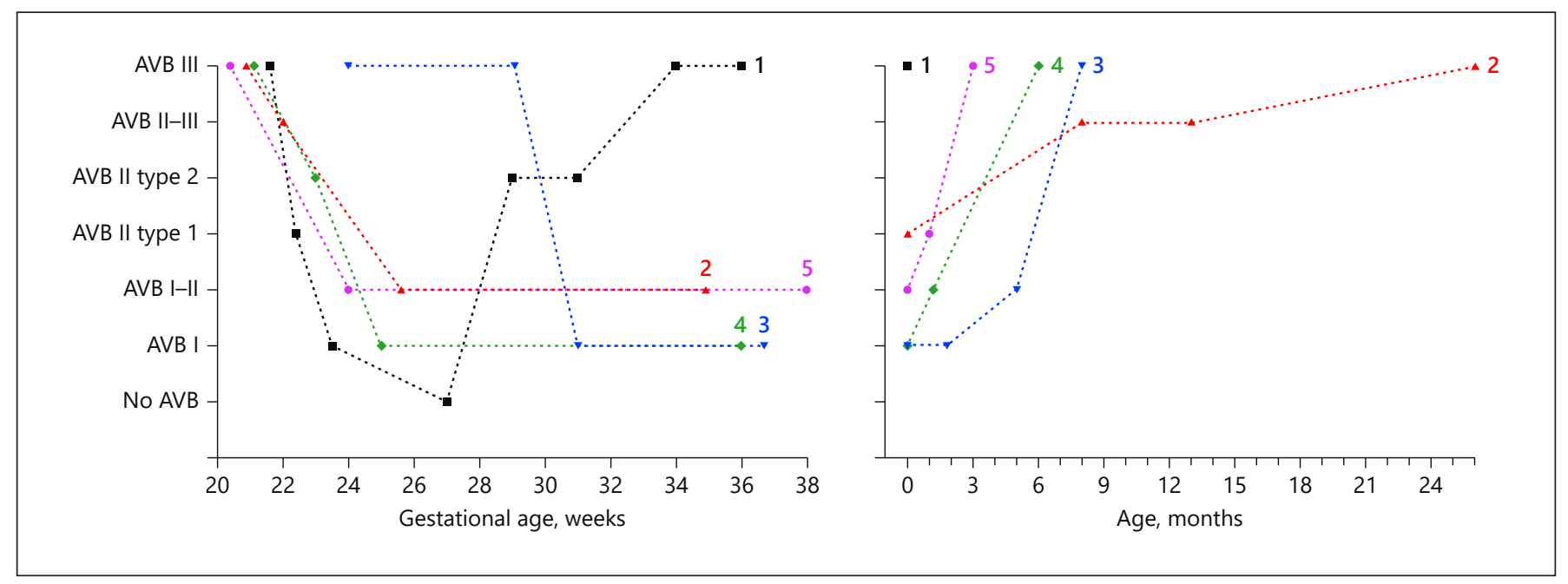

Fig. 1. Prenatal (left panel) and postnatal (right panel) evolution in AV conduction of the 5 treatment responders (cases 1-5; see result section for details). AVB, atrioventricular block; III, third-degree or complete; II, seconddegree; I, first-degree; AV, atrioventricular.

Table 1. Characteristics of study cases without (nonresponders) versus with (responders) improvement in AV conduction on transplacental dexamethasone treatment

\begin{tabular}{|c|c|c|c|c|c|}
\hline Variables & Nonresponders & & Responders & & $p$ values \\
\hline$n$ & 54 & & 5 & & \\
\hline CAVB diagnosis, weeks & $23(17-33)$ & & $21(20.4-24)$ & & 0.18 \\
\hline Heart rate at diagnosis, bpm & $58(38-92)$ & & $80(60-97)$ & & 0.0036 \\
\hline Heart rate $>70 \mathrm{bpm}, n(\%)$ & $5(9)$ & & $4(80)$ & & 0.0013 \\
\hline EFE at diagnosis, $n(\%)$ & $12 / 54(22)$ & & $2 / 5(40)$ & & 0.58 \\
\hline Prenatal dexamethasone, $n(\%)$ & $54 / 54(100)$ & & $5 / 5(100)$ & & \\
\hline Start of treatment, weeks & $24(17-33)$ & & $21(20.4-24)$ & & 0.12 \\
\hline Starting dose & $\leq 24$ weeks & $>24$ weeks & $\leq 24$ weeks & $>24$ weeks & \\
\hline $4 \mathrm{mg} /$ day & 5 & 13 & 0 & 0 & \\
\hline $8 \mathrm{mg} /$ day & 24 & 12 & 5 & 0 & \\
\hline Duration of treatment, weeks & $11.5(2-18)$ & & $15(12.9-17.7)$ & & 0.04 \\
\hline Prenatal IVIG, $n(\%)$ & $10 / 54(20)$ & & $3 / 5(60)$ & & 0.067 \\
\hline Start of treatment, weeks & $20.5(17-25)$ & & $23(20.4-26)$ & & 0.21 \\
\hline Prenatal salbutamol, $n(\%)$ & $20 / 54(37)$ & & $0 / 5(0)$ & & 0.16 \\
\hline Start of treatment, weeks & $30(20-35)$ & & - & & \\
\hline Duration of treatment. weeks & $5(1-11)$ & & - & & \\
\hline Oligohydramnios, $n(\%)$ & $6 / 54(11)$ & & $1 / 5(20)$ & & 0.48 \\
\hline Age at delivery, weeks & $36.7(23-39)$ & & $36.0(36.0-38.1)$ & & 0.51 \\
\hline Weight at delivery, kg & $2.49(0.31-3.4)$ & & $2.27(1.61-2.47)$ & & 0.75 \\
\hline Permanent pacemaker implant, $n(\%)$ & $45 / 49(92)$ & & $2 / 5(40)$ & & 0.013 \\
\hline Follow-up, years & $10.3(1.1-18)$ & & $12.3(1.5-16.5)$ & & 0.69 \\
\hline
\end{tabular}

bpm, beats per minute; CAVB, CAVB, complete atrioventricular block; EFE, endocardial fibroelastosis; IVIG, intravenous immune globulin; AV, atrioventricular. Median (range) or $n$ (\%). Fisher's exact test for categorical variables and Student's $t$ test or Mann-Whitney U test for continuous variables were employed, as appropriate. 


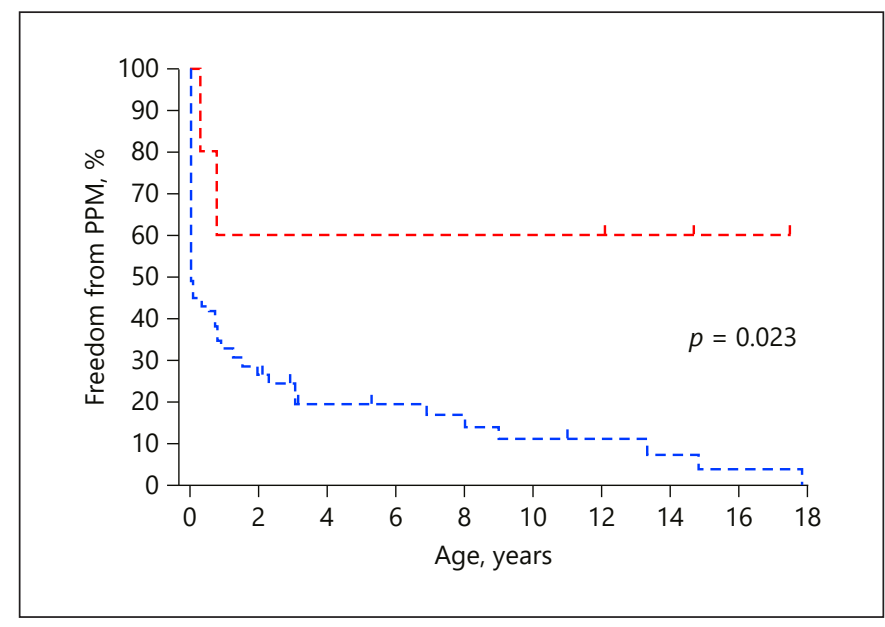

Fig. 2. Kaplan-Meier freedom of pacemaker implantation comparing nonresponders (blue line) and responders (red line) to prenatal CAVB treatment with dexamethasone ( $\log$ rank, $\chi^{2}=5.19$; $p=0.023)$. CAVB, complete atrioventricular block.

weeks despite high-dose dexamethasone ( $6 \mathrm{mg}$ /day) and IVIG and to CAVB at 34 weeks. At 17.5 years, this female is asymptomatic and unpaced (average VR $51 \mathrm{bpm}$ ).

Case 2 presented with CAVB (VR $97 \mathrm{bpm}$ ) at 21 weeks gestation. On dexamethasone, this improved to intermittent type-2 AVB II at 22 weeks and to type-1 AVB II from $25+4$ weeks to delivery. The girl was delivered at $34+6$ weeks for oligohydramnios when type-1 AVB II was confirmed by ECG. Holter at 13 and 26 months of life, respectively, showed predominant and finally permanent CAVB. At 15 years of life, she is well and unpaced (average VR $59 \mathrm{bpm}$ ).

Case 3 was referred at 24 weeks for fetal CAVB (VR 60 $\mathrm{bpm})$ and ascites. The effusion resolved with dexamethasone but CAVB continued to 29 weeks when the steroid treatment was reduced to $2 \mathrm{mg} /$ day as per protocol. At 31 weeks, AVB I (AVI $220 \mathrm{~ms}$ ) with a heart rate of $135 \mathrm{bpm}$ was noted. The boy was electively delivered at $36+5$ weeks and AVB I (PR interval $184 \mathrm{~ms}$ ) confirmed by ECG. Holter at 5 months of life showed intermittent AVB 2 with an average heart rate of $82 \mathrm{bpm}$. He presented with decreased appetite and lethargy at 8 months when severe ventricular dysfunction and CAVB (VR $46 \mathrm{bpm}$ ) were noted for which he required an emergency pacemaker implant. He is well at 12.3 years of life with normalized cardiac function.

Case 4 was diagnosed with CAVB (VR 95 bpm) and echogenic AV valvar papillary muscles at 21 weeks. On dexamethasone and IVIG, 2:1 AVB was documented at 23 weeks that had improved to AVB I (AVI $220 \mathrm{~ms}$ ) 2 weeks later. AVB I (PR interval $216 \mathrm{~ms}$ ) was confirmed after the girl's delivery at 36 weeks. She was well until 6 months of life when she presented in heart failure secondary to spontaneously ruptured tricuspid valvar chordae and a new diagnosis of CAVB (VR $94 \mathrm{bpm}$ ). The tricuspid valve was successfully repaired. She is asymptomatic and unpaced (VR $48 \mathrm{bpm}$ ) at 12.1 years of life.

Case 5 presented at $20+3$ weeks with CAVB (VR 75 bpm), EFE, and runs of ventricular tachycardia up to 255 bpm. On dexamethasone and IVIG, the tachycardia resolved. Variable AV conduction (AVB I, type-1, and type-2 AVB II) with heart rates at around $110 \mathrm{bpm}$ was documented from 24 weeks to birth at 38 weeks. The AV conduction deteriorated to $2: 1 \mathrm{AVB}$ at 1 month and CAVB (VR $54 \mathrm{bpm}$ ) at 3 months when the girl underwent elective epicardial pacemaker implant. She remains well at 1.5 years of life.

\section{Outcome of Nonresponders}

Of 54 nonresponders, 3 cases died in utero and 2 after birth following withdrawal of care (extreme prematurity; asphyxia). Table 1 and Figure 2 illustrate the differences in timing of postnatal pacing between responders and actively managed nonresponders. The median age at pacemaker implant of nonresponders was 5 days.

\section{Discussion}

The current understanding of the pathomechanism of immune-mediated CAVB includes that the deposition of maternal anti-Ro antibodies in the fetal heart may initiate a cascade of inflammatory responses that in turn contribute to the fibrosis and calcification of the AV node. This process can begin as early as 16-18 gestation weeks when increasing amounts of maternal IgG cross the placenta, bind apoptotic cardiocytes, and block their physiological removal $[12,13]$. The ensuing inflammation may then initially manifest as a delay or partial blockage of the atrioventricular electrical impulse propagation and still be reversible with transplacental steroids. Indeed, we and others $[4,5]$ have shown that prenatal anti-inflammatory treatment of incomplete AVB may persistently improve or normalize the AV conduction. Nonetheless, incomplete fetal heart block is a rare observation probably due to a rapid transition from normal $\mathrm{AV}$ conduction to CAVB within less than a week $[5,14]$.

Once established, CAVB is considered as the clinical manifestation of irreversible AV nodal damage. This concept is supported by limited pathology data in the medical 
literature. Llanos et al. [3] reviewed the postmortem findings of a handful of fetuses that were diagnosed with CAVB several weeks before the fetal demise. Extensive fibrosis/calcification of the AV node \pm the bundle of his in the absence of residual inflammation was documented in all specimens. Presumably related to a similarly advanced pathology, none of our study cases diagnosed with CAVB after 24 gestation weeks responded to anti-inflammatory medication with an improvement in AV conduction. Our findings, however, support that high-dose dexamethasone may restore AV conduction if treatment is initiated at an earlier gestational age, arguably closer to the disease onset, and if the VR is faster than usual for fetal CAVB. Indeed, sustained reversibility of CAVB with treatment was exclusively observed in cases treated $\leq 24$ weeks and all but 1 responder displayed baseline VRs above $70 \mathrm{bpm}$. Supporting the concept of a functional conduction blockage due to tissue inflammation in these cases, AV conduction partially resumed within a few days of treatment (Fig. 1) and then continued to further improve over the next 2-5 weeks to birth. In case 3 , however, diagnosed later (24 weeks) and with a lower VR $(60 \mathrm{bpm})$ compared with the other responders, CAVB persisted for $>6$ weeks until AVB I was first documented. This observation cannot be solely explained by an anti-inflammatory treatment effect but implies other mechanisms to play a role, such as the new formation of viable fetal AV conduction tissue.

Despite the perinatal use of anti-inflammatory medication, CAVB eventually reappeared in all responders either before or after birth: this outcome, however, was associated with a significantly lower requirement of postnatal pacing when compared with nonresponders (Fig. 2). Mechanisms to explain the perinatal progression from incomplete to complete heart block are speculative but likely include ongoing AV nodal scarring and the physiologic loss of cardiocyte proliferation after birth. Future treatment strategies to preserve AV conduction beyond birth may therefore include the addition of anti-fibrotic medication.

\section{Limitations}

As outlined in the method section, dexamethasone starting doses depended on the era of CAVB diagnosis. While our findings support the potential of CAVB reversal with $8 \mathrm{mg} /$ day before 24 weeks, there are insufficient case numbers $(n=5)$ in this age-group to assess the impact of the high versus low dose steroids. Regression of CAVB with $4 \mathrm{mg} /$ day has, however, not been reported in the literature. It is also not possible to conclude on the role of IVIG in this study: although a larger proportion of re- sponders were prenatally treated with IVIG (Table 1), only $2 / 5$ cases did receive a first dose of this medication at the time of CAVB diagnosis.

In summary, transplacental dexamethasone restored AV conduction in a subgroup of fetuses with immunemediated CAVB. This temporary effect was associated with a lower rate of postnatal pacing when compared with the cohort of nonresponders.

\section{Statement of Ethics}

This study was approved by the SickKids Research Ethics Board (REB\# 1000011904). Study procedures were conducted in accordance with the World Medical Association Declaration of Helsinki. The need of written parental consent of patient enrollment was waived by the IRB.

\section{Conflict of Interest Statement}

The authors do not have any commercial interest or other association that might pose a conflict of interest, and they are independent from funders and sponsors.

\section{Funding Sources}

Dr. Saito received research funds from (a) the Sakakibara Clinical Research for Promotion of Sciences, Japan, and (b) the Labatt Family Heart Center, Toronto, Canada.

\section{Author Contributions}

All authors were involved in the drafting of the manuscript. All provided approval of the version to be published and agreed to be accountable for all aspects of the work. In addition to this, all authors further contributed to the undertaking of the study. F.G., V.G., V.T., and E.J. were involved in the design and conception of the work. M.S., F.G., and E.J. contributed to acquisition, analysis, and interpretation of the data. E.S., F.G., V.G., L.H., and V.T. provided substantial contributions to the revision of the work and interpretation of the results.

\section{References}

1 Jaeggi E, Laskin C, Hamilton R, Kingdom J, Silverman $\mathrm{E}$. The importance of the level of maternal anti-Ro/SSA antibodies as a prognostic marker of the development of cardiac neonatal lupus erythematosus a prospective study of 186 antibody-exposed fetuses and infants. J Am Coll Cardiol. 2010 Jun 15;55(24):2778-84.

2 Ho SY, Esscher E, Anderson RH, Michaëlsson M. Anatomy of congenital complete heart block and relation to maternal anti-Ro antibodies. Am J Cardiol. 1986 Aug 1;58(3):291-4. 
3 Llanos C, Friedman DM, Saxena A, Izmirly PM, Tseng CE, Dische R, et al. Anatomical and pathological findings in hearts from fetuses and infants with cardiac manifestations of neonatal lupus. Rheumatology. 2012 Jun; 51(6):1086-92.

4 Saleeb S, Copel J, Friedman D, Buyon JP. Comparison of treatment with fluorinated glucocorticoids to the natural history of autoantibody-associated congenital heart block: retrospective review of the research registry for neonatal lupus. Arthritis Rheum. 1999 Nov;42(11):2335-45.

5 Kan N, Silverman ED, Kingdom J, Dutil N, Laskin C, Jaeggi E. Serial echocardiography for immune-mediated heart disease in the fetus: results of a risk-based prospective surveillance strategy. Prenat Diagn. 2017 Apr;37(4): 375-82.

6 Carvalho JS, Shinebourne EA, Kyle P. Efficacy unproved of maternal dexamethasone for fetal heart block. Am J Obstet Gynecol. 1996 Aug;175(2):502-3.

7 Jaeggi ET, Fouron JC, Silverman ED, Ryan G, Smallhorn J, Hornberger LK. Transplacental fetal treatment improves the outcome of prenatally diagnosed complete atrioventricular block without structural heart disease. Circulation. 2004 Sep 21;110(12):1542-8.
8 Nii M, Hamilton RM, Fenwick L, Kingdom JC, Roman KS, Jaeggi ET. Assessment of fetal atrioventricular time intervals by tissue Doppler and pulse Doppler echocardiography: normal values and correlation with fetal electrocardiography. Heart. 2006 Dec;92(12):1831-7.

9 Gregoratos G, Cheitlin MD, Conill A, Epstein AE, Fellows C, Ferguson TB, et al. ACC/AHA guidelines for implantation of cardiac pacemakers and antiarrhythmia devices: executive summary. A report of the American College of Cardiology/American Heart Association task force on practice guidelines (committee on pacemaker implantation). Circulation. 1998 April 97:1325-35.

10 Epstein AE, DiMarco JP, Ellenbogen KA, Estes NA 3rd, Freedman RA, Gettes LS, et al. ACC/AHA/HRS 2008 guidelines for devicebased therapy of cardiac rhythm abnormalities: a report of the American College of Cardiology/American Heart Association task force on practice guidelines (writing committee to revise the ACC/AHA/NASPE 2002 guideline update for implantation of cardiac pacemakers and antiarrhythmia devices): developed in collaboration with the American Association for Thoracic Surgery and Society of Thoracic Surgeons. Circulation. 2008 May 27;117(21):e350-408.
11 Jaeggi ET, Silverman ED, Yoo SJ, Kingdom J. Is immune-mediated complete fetal atrioventricular block reversible by transplacental dexamethasone therapy? Ultrasound Obstet Gynecol. 2004 Jun;23(6):602-5.

12 Clancy RM, Kapur RP, Molad Y, Askanase $\mathrm{AD}$, Buyon JP. Immunohistologic evidence supports apoptosis, IgG deposition, and novel macrophage/fibroblast crosstalk in the pathologic cascade leading to congenital heart block. Arthritis Rheum. 2004 Jan;50(1):17382.

13 Briassouli P, Komissarova EV, Clancy RM, Buyon JP. Role of the urokinase plasminogen activator receptor in mediating impaired efferocytosis of anti-SSA/Ro-bound apoptotic cardiocytes: Implications in the pathogenesis of congenital heart block. Circ Res. 2010 Aug 6;107(3):374-87.

14 Cuneo BF, Sonesson SE, Levasseur S, MoonGrady AJ, Krishnan A, Donofrio MT, et al. Home monitoring for fetal heart rhythm during anti-Ro pregnancies. J Am Coll Cardiol. 2018 Oct 16;72(16):1940-51. 